\title{
UCRL-TR-208078
}

LAWRENCE LIVERMORE N A T IO N A L LABORATORY

\section{Comparison of Streak Tube Performance}

R. A. Lerche, D. S. Andrews, P. M. Bell, R. L. Griffiths, A. W. Huey, J. W. McDonald, G. Vergel de Dios

November 17, 2004 
This document was prepared as an account of work sponsored by an agency of the United States Government. Neither the United States Government nor the University of California nor any of their employees, makes any warranty, express or implied, or assumes any legal liability or responsibility for the accuracy, completeness, or usefulness of any information, apparatus, product, or process disclosed, or represents that its use would not infringe privately owned rights. Reference herein to any specific commercial product, process, or service by trade name, trademark, manufacturer, or otherwise, does not necessarily constitute or imply its endorsement, recommendation, or favoring by the United States Government or the University of California. The views and opinions of authors expressed herein do not necessarily state or reflect those of the United States Government or the University of California, and shall not be used for advertising or product endorsement purposes.

This work was performed under the auspices of the U.S. Department of Energy by University of California, Lawrence Livermore National Laboratory under Contract W-7405-Eng-48. 


\title{
COMPARISON OF STREAK TUBE PERFORMANCE
}

\author{
R. A. Lerche, D. S. Andrews, P. M. Bell, R. L. Griffith, \\ A. W. Huey, J. W. McDonald, and G. Vergel de Dios \\ Lawrence Livermore National Laboratory, 7000 East Avenue, Livermore, CA 94551
}

\begin{abstract}
The performance of four streak tubes in six streak camera configurations is reported. Evaluations were made as part of a search for a streak tube to replace the obsolete RCA C73435 used in the ICF Program's optical streak cameras. Characteristics measured include linearity, spatial and temporal resolution, line-spread function, contrast transfer ratio (CTR), and dynamic range. Tubes evaluated are the RCA C73435, Photonis P510, Photek ST-Y, and Hamamatsu N8059. The RCA C73435 was evaluated in three camera configurations: large format CCD coupled directly to the streak tube, CCD directly coupled to an image intensifier tube (IIT), and the original configuration with a smaller CCD lens coupled to the IIT output. The Photonis and Photek tubes were characterized in configurations where they were directly coupled to large format CCDs. Optimum spatial resolution is achieved when the IIT is removed. Maximum dynamic range requires a configuration where a single photoelectron from the photocathode produces a signal that is $\sim 5$ times the CCD read noise. The Photonis P510 tube with the E2V CCD forms a well-optimized streak camera system.
\end{abstract}

\section{INTRODUCTION}

The Lawrence Livermore National Laboratory (LLNL) Laser Programs built more than 30 of its high-speed optical streak cameras ${ }^{1}$ in the 1970s and 1980s. These cameras have provided the Inertial Confinement Fusion (ICF) Program with high-speed recording capability for nearly 30 years. The cameras are used as the recording device for a variety of laser $^{2}, \mathrm{x}-\mathrm{ray}^{3}$, and particle instruments ${ }^{4}$ used in experiments that require temporal resolutions approaching 10 picoseconds. The cameras use a large format (40-mm diam) RCA C73435 streak tube directly coupled to a microchannel-plate (MCP) image-intensifier tube (IIT). Before 1987, images were captured on hard-film at the output of the IIT. In the mid 1980s, 15 cameras were built for the Nova laser project. These incorporated CCD readouts in place of the earlier film packs ${ }^{5}$. Coupling between the IIT and the CCD was done with a lens system with a reduction ratio of $\sim 2.5: 1$. Lens coupling was selected because direct fiber-optic coupling was not yet reliable.

While the original optical streak cameras have worked extremely well for the ICF Program, they are, unfortunately, becoming difficult to maintain. The streak tube, the IIT, and several other components are no longer commercially available. In 1997 a replacement CCD was identified that has extended the life of the LLNL cameras by eight years . The projected need of approximately 50 optical streak cameras for the National Ignition Facility (NIF) requires identification of replacement streak tubes for existing cameras and the acquisition of additional cameras. The work described in this report is related to the search for a streak tube that can replace the RCA C73435 in current LLNL optical streak cameras and that can be used in future cameras.

In this study we evaluated six streak camera configurations and four streak tubes. We first characterized the standard LLNL "blue box" streak camera (named for its LLNL "laser blue" color). Since it has been used by LLNL scientists and engineers for 30 years, its performance is well known to many users and provides an excellent benchmark against which the other cameras and tubes can be compared. Next, two variations of the LLNL camera were studied to demonstrate the impact of the MCP IIT on camera resolution and the potential advantage of using a modern, large format CCD as the instrument readout. By using a large-format CCD directly coupled to the output of the streak tube, it is possible to eliminate the IIT and the lens coupling to the CCD. It has long been known, that the MCP IIT degrades spatial performance of the streak camera ${ }^{7}$, thus its elimination can improve spatial resolution. A Photek ST-Y streak tube was evaluated in a slightly modified LLNL streak camera. In addition, a Photonis P510 streak tube was evaluated in a prototype camera jointly developed by the Laboratory for Laser Energetics (LLE) at the University of Rochester and LLNL that Livermore calls the Generic Streak Camera Platform (GSCP) and Rochester calls the ROSS (for Rochester Optical Streak System). We evaluated a ROSS version of the camera. A magnetically focused Hamamatsu 7700 camera 
with an N8059 streak tube was also evaluated. Both the Photek and Photonis tubes can be adapted to the LLNL camera body. The Hamamatsu camera represents an alternate camera choice that has been purchased by several groups at LLNL.

This report begins with a description of the six streak-camera configurations studied, followed by descriptions of the characterization parameters and measurement techniques, and a discussion of the results. Portions of this report also appear in references [8 and 9]

\section{CAMERA CONFIGURATIONS}

Three streak camera configurations characterized in this work are based on the RCA C73435 streak tube (see Fig. 1 and Table 1). The basic "blue box" camera, which was developed during the 1970s, included a $40-\mathrm{mm}$, proximity focused ITT F4113 MCP IIT directly coupled to the streak tube for signal amplification, and used contact film for recording the output image. Circuit improvements and a CCD readout were added in the $1980 \mathrm{~s}^{5}$. The original CCD readout was a Photometrics camera incorporating a Thompson CSF TH-7861 CCD, a 576 X 384 array of 24-- $7 \mathrm{~m}$ square pixels. The CCD was lens coupled to the IIT with an image-size reduction of approximately 2.5:1. In 1996, the ICF Program began replacing these obsolete CCD readouts with Photometrics SenSys 1600 CCD cameras containing Kodak KAF 1600 CCDs that have $1534 \times 1024$ arrays of $9-\square \mathrm{m}$ pixels. ${ }^{6}$ The Kodak CCD has properties very similar to the original Thompson CCD. It is exactly the same size, its sensitivity of $20 \mathrm{e}-/ \mathrm{ADU}$ is similar to the original $25 \mathrm{e}-/ \mathrm{ADU}$, and with $3 \times 3$ binning the $27-\square \mathrm{m}$ super-pixels are a close match to the original $24 \square \mathrm{m}$ pixels. The newer readout works with the same lens coupling design as the earlier CCD.

Detailed characterization of the LLNL streak camera in the late 1980s established optimum operating parameters that are still used today. Two of the most significant operating parameters are the MCP IIT gain ${ }^{10}$ and the input slit configuration $^{11}$. The IIT luminous gain is routinely set at 4,000. At this gain, signal from a single photoelectron is well matched to the noise characteristics of the streak camera to provide maximum dynamic range. The standard streak camera input consists of an external slit whose image is optically relayed to the photocathode of the streak tube. Originally, the slit width was set at $100 \square \mathrm{m}$. During characterization work, it was discovered that the parallel wire structure of the extraction grid in the streak tube allowed electrostatic focusing of a wide input slit down to a fine output line comparable to the output achieved with a $100-\square \mathrm{m}$ wide slit. Standard camera setup includes setting the input slit width to $1000 \square \mathrm{m}$. This provides the camera with two advantages: A factor of 10 increase in useful photocathode area and thus a factor of 10 increase in sensitivity to low-level signals, and the photocathode current density is reduced by a factor of 10 to achieve the same amplitude output signal.

Besides its standard setup, the LLNL camera was reconfigured with the RCA C73435 tube directly coupled to a large format SI-800 CCD to demonstrate the potential of the RCA C73435 tube without the degradation in spatial resolution caused by the MCP IIT. Finally, the LLNL camera was reconfigured with a MCP IIT directly coupled to a large format CCD to emphasize the effect of the MCP IIT.

(a)

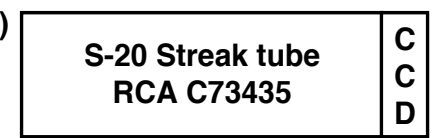

(b)

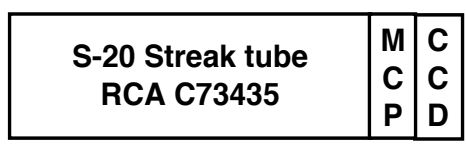

(c)

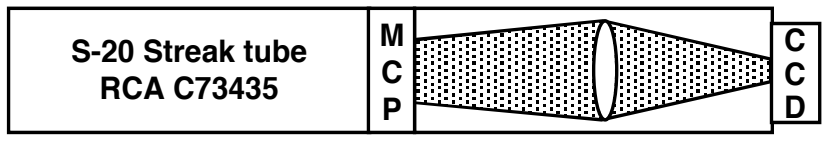

Figure 1. Streak camera configurations tested. 
Table 1. List of streak tubes and streak camera configurations tested.

\begin{tabular}{|l|l|l|l|l|}
\hline $\begin{array}{l}\text { Streak Camera } \\
\text { Configuration }\end{array}$ & Streak Tube & Image Intensifier & $\begin{array}{l}\text { Coupling } \\
\text { To CCD }\end{array}$ & CCD \\
\hline 1. LLNL - Fig. 1(c) & RCA C73435 & ITT F4113 & Lens & Kodak KAF1600 \\
\hline 2. LLNL - Fig. 1(a) & RCA C73435 & None & Direct & Kodak KAF16800 \\
\hline 3. LLNL - Fig. 1(b) & RCA C73435 & ITT F4113 & Direct & Kodak KAF16800 \\
\hline 4. LLNL - like Fig 1(a) & Photek ST-Y & None & Direct & Kodak KAF16800 \\
\hline 5. ROSS - like Fig 1(a) & Photonis P510 & None & Direct & E2V 42-40 BI \\
\hline 6. Hamamatsu 7700 & Hamamtsu N8059 & Hamamatsu N8059 & Lens & C4880-21-24A \\
\hline
\end{tabular}

Kodak KAF1600 - 1536X1024 array of 9 mm pixels in Photometrics SenSYS 1600 CCD camera. Kodak KAF16800 - 4096X4096 array of 6 mm pixels in Spectral Instruments SI800 camera E2V 42-40 BI - 2048X2048 array of $13.5 \mathrm{~mm}$ back illuminated pixels in SI800 camera Hamamatsu C4880-21-24A - 1024X1024 array

Three other streak tubes were also characterized, each in a different camera configuration. A Photek ST-Y was evaluated in a modified LLNL camera. The Photek tube replaced the RCA C73435 and the large format CCD was coupled directly to the streak tube's output, similar to the configuration in Fig. 1(a). A Photonis P510 streak tube was characterized in the highly optimized ROSS prototype version of LLNL's GSCP camera. It has the streak tube directly coupled to a large format, low noise E2V CCD through a low-distortion 1:1 fiber-optic coupler. The Photonis P510 can also be retrofitted into the LLNL "blue box". Since LLNL had recently purchased three Hamamatsu 7700 cameras, one of these cameras was also characterized. The Hamamatsu has a smaller diameter streak tube, an image intensifier stage, and a configuration similar to Fig. 1(c).

\section{STREAK CAMERA CHARACTERIZATION}

Characterization is the process of determining how a streak camera performs for a variety of operating conditions. It is done to determine the optimum component configuration and to select their best operating points. Characterization tests reported for the optical cameras evaluated in this study fall into the following categories:

1. Streak tube sensitivity,

2. Spatial resolution,

3. Temporal resolution,

4. Background measurements,

5. System sensitivity measurements, and

6. Dynamic range.

Unless noted otherwise, reported measurements used a 1-mm wide input slit illuminated with a collimated 532-nm laser. Collimated light passing through the input optics of a camera has a large depth of field at the streak camera photocathode which helps eliminate the input optic as a factor in resolution measurements.

\subsection{Streak tube sensitivity and current limit}

Measurement of a streak tube's sensitivity and a calculation of its Child-Langmuir (C-L) space-charge-limited current are excellent starting points for streak camera characterization. These are two fundamental characteristics that are related only to the streak tube. The C-L current ultimately determines the upper limit of a streak camera's dynamic range.

Sensitivity, which is typically measured in units of amps/watt as a function of incident light wavelength, relates photocathode current to the optical power incident on the photocathode. A calibrated DC light source is used to measure sensitivity. The light source illuminates the streak tube through a set of narrow-band filters at various wavelengths and the corresponding photocathode current is measured. Streak tube electrodes (the grids, deflection plates, and anode) are 
electrically connected together and biased at +300 volts relative to the photocathode to collect all photoelectrons emitted from the photocathode. Since streak cameras are used to observe relatively short duration pulses of light, it is often convenient to think of sensitivity in units of Coulombs/Joule. Typical sensitivities for the RCA C73435 with S-1 and S20 photocathodes at typical ICF laser wavelengths are listed in Table 2. Tubes from other manufacturers have similar sensitivities for similar photocathode materials.

\begin{tabular}{|c|c|c|c|c|}
\hline Photocathode & Wavelength & $\mathrm{S}$ (ma/watt) & $\mathrm{P}_{\mathrm{J} 0}\left(\mathrm{watt} / \mathrm{cm}^{2}\right)$ & $\mathrm{E}_{\mathrm{J} 0}\left(\mathrm{~nJ} / \mathrm{cm}^{2}\right)$ \\
\hline \multirow[t]{3}{*}{ S-1 } & $1.06 \mu \mathrm{m}$ & 0.4 & 1,064 & 107 \\
\hline & $0.53 \mu \mathrm{m}$ & 0.8 & 503 & 50 \\
\hline & $0.35 \mu \mathrm{m}$ & 4.5 & 94 & 9 \\
\hline \multirow[t]{2}{*}{ S-20 } & 0.53 & 50.0 & 8 & 0.8 \\
\hline & $0.35 \mu \mathrm{m}$ & 40.0 & 11 & 1.1 \\
\hline
\end{tabular}

The maximum useful tube current (or maximum useful charge in a short pulse) is proportional to the C-L space-chargelimited current. This quantity depends on the internal geometry of the streak tube and the bias potentials applied to the electrodes. For the RCA, Photonis, and Photek tubes, the photocathode and extraction grid closely approximate a parallel plate photodiode (see Fig. 2). The C-L current in this configuration is given by:

$$
\mathrm{J}_{0}=(4 \square / 9)(2 \mathrm{e} / \mathrm{m})^{1 / 2}\left(\mathrm{~V}^{3 / 2} / \mathrm{x}^{2}\right),
$$

where $\mathrm{J}_{0}$ is the C-L space-charge-limited current density $\left(\mathrm{amps} / \mathrm{cm}^{2}\right)$, $\square$ is the emissivity in vacuum $(1 / 36 \square) \times 10^{9}$ coulomb, $\mathrm{e}$ is the charge of an electron, $\mathrm{m}$ is the mass of an electron, $\mathrm{x}$ is the electrode separation, and $\mathrm{V}$ is the potential applied between photocathode and extraction grid. For the RCA C73435, $\mathrm{x}$ and V are $7 \mathrm{~mm}$ and 2500 volts, respectively, and for the Photonis P510 they are $3.5 \mathrm{~mm}$ and 2500 volts. Because the extraction grid is formed by a slot (or a set of parallel wires $5 \mathrm{~mm}$ apart), rather than a tight mesh used in many other streak tubes, the electric field at the photocathode is reduced slightly below that of extraction grid. The C-L limit at 2200 volts is $\sim 0.5 \mathrm{amps} / \mathrm{cm}^{2}$ for the $\mathrm{C} 73435$ and $\sim 2$ amps $/ \mathrm{cm}^{2}$ for the P510. This simple calculation shows the P510 can handle 4 times more current density than the C73435. The laser intensity required to reach this current is given by:

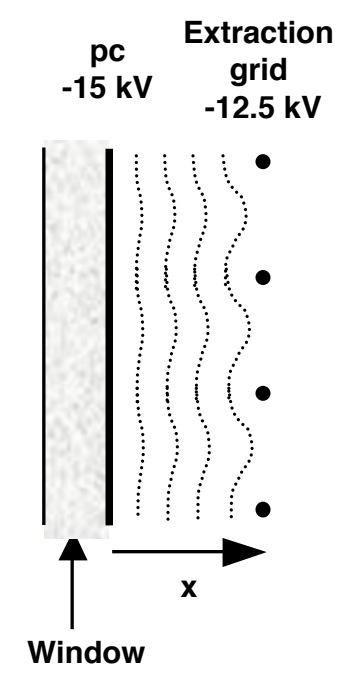

Figure 2. Photocathode to extraction-grid geometry of RCA C73435. 


$$
\mathrm{P}_{\mathrm{J} 0}=\mathrm{J}_{0} / \mathrm{S},
$$

where $\mathrm{S}$ is the photocathode sensitivity. The required laser fluence needed to reach the $\mathrm{C}-\mathrm{L}$ current limit is

$$
\mathrm{E}_{\mathrm{J} 0}=\mathrm{P}_{\mathrm{J} 0} \square \mathrm{t}
$$

where $\square \mathrm{t}$ is the pulse length. Table 2 also lists the laser intensity $\mathrm{P}_{\mathrm{J} 0}$ and pulse fluence $\mathrm{E}_{\mathrm{J} 0}$ needed to reach the C-L space-

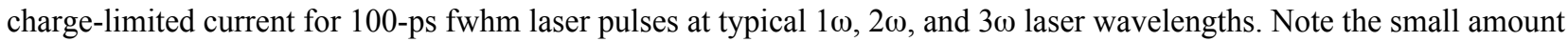
of energy needed to reach the C-L current limit. Typically, a couple percent of the C-L limit (a few pJ into an S-20 photocathode) will cause noticeable saturation effects in the tube. (See Ref. 12 for a detailed discussion of the C-L current.)

The Hamamatsu N8059 has a mesh-type extraction grid instead of a slot like the other tubes tested and is magnetically focused. Electrostatic lenses formed by the slot and the focus grid in the other tubes can focus electrons from a relatively wide strip $(0.5$ to $1 \mathrm{~mm})$ on the photocathode to a narrow line at the tube's output. ${ }^{11}$ Because image width for a meshtype tube is proportional to the width of the illuminated strip, its input slit is typically $<100 \square \mathrm{m}$ wide to maintain good time resolution. Thus, a slot-type tube can have 10 times more usable photocathode area, 10 times more sensitivity, and support 10 times more current than a mesh-type tube with similar extraction grid voltage and spacing. We found the useful photocathode strip width to be $1 \mathrm{~mm}$ and $500 \mathrm{~mm}$ for the RCA C73435 and the Photonis P510, respectively. Mesh grids typically have a lower throughput as the screen intercepts 25 to $40 \%$ of the photoelectrons. The magnetically focused Hamamatsu tube uses a higher extraction voltage, so should support a higher C-L current density. Needed internal structure data was not available to calculate the C-L current for the Hamamatsu or Photek tubes.

\subsection{Spatial resolution}

A line-spread function (LSF) and a contrast transfer function (CTF) are alternate ways to describe spatial resolution. In this work we measured the system LSF, then calculated the CTF by convolving the LSF with square waves of various frequencies. Then to test the validity of the calculation, the CTF was measured using Ronchi ruling masks at appropriate spatial frequencies (typically 4, 6, and $10 \mathrm{lp} / \mathrm{mm}$ ).

We begin spatial resolution measurements with a determination of the system spatial magnification between the streak tube photocathode and the CCD read out. This is done by illuminating a mask of parallel $10-\mu \mathrm{m}$ wide slits spaced 1.5 $\mathrm{mm}$ apart along the input slit of the streak camera. Next, we illuminate a single $10-\mu \mathrm{m}$ wide spatial slit with an 18 -ns long laser pulse. The measured LSF is simply signal amplitude versus spatial position. Typically, the LSF for each camera is measured at a variety of signal amplitudes to demonstrate linearity over a wide range of signal amplitudes.

A sample LSF data image is shown in Fig. 3(a); normalized LSF signals from the six camera configurations are overlaid in Fig. 3(b). The spatial resolution of the Photonis P510 and the RCA C73435 (without IIT) show excellent spatial resolution of 40 and $50 \square \mathrm{m}$ (fwhm), respectively, referred to the streak tube photocathode. This is about a factor of three better than the $120 \square \mathrm{m}$ fwhm of the standard LLNL "blue box". The LSF of the P510 is a sharp spike rising a factor of $\sim 500$ above the noise. The RCA C73435 (without IIT) also shows a sharp narrow spike, but has significant wings in the LSF at $\sim 2 \%$ of the peak intensity. The remaining three configurations have LSFs similar to the standard LLNL camera. In the Hamamatsu and LLNL (configuration 3) the IIT limits the spatial resolution at $\sim 100 \square \mathrm{m}$ fwhm and produces wings on the LSF characteristic of veiling glare in a MCP IIT. Since the Photek ST-Y (configuration 5) is not coupled to a MCP IIT, it is concluded that the measured response in this case is that of the streak tube.

Figure 4 shows the CTFs calculated by convolving square waves with each of the six LSFs presented in Fig. 3. The ROSS configuration shows an excellent CTF that is flat to $4 \mathrm{lp} / \mathrm{mm}$ and shows a limiting visual resolution (5\% CTF) greater than $25 \mathrm{lp} / \mathrm{mm}$. The RCA C73435 streak tube directly coupled to a large format CCD also shows good high frequency performance with a limiting visual resolution of $20 \mathrm{lp} / \mathrm{mm}$. This configuration, however, shows degraded performance between 2 and $7 \mathrm{lp} / \mathrm{mm}$ that is caused by the low level wings on the LSF. The cause of the low level wings for this camera configuration was not investigated. The standard LLNL, Photek, and Hamamatsu cameras produced significantly poorer spatial resolution with the Hamamatsu being the best of these three. 

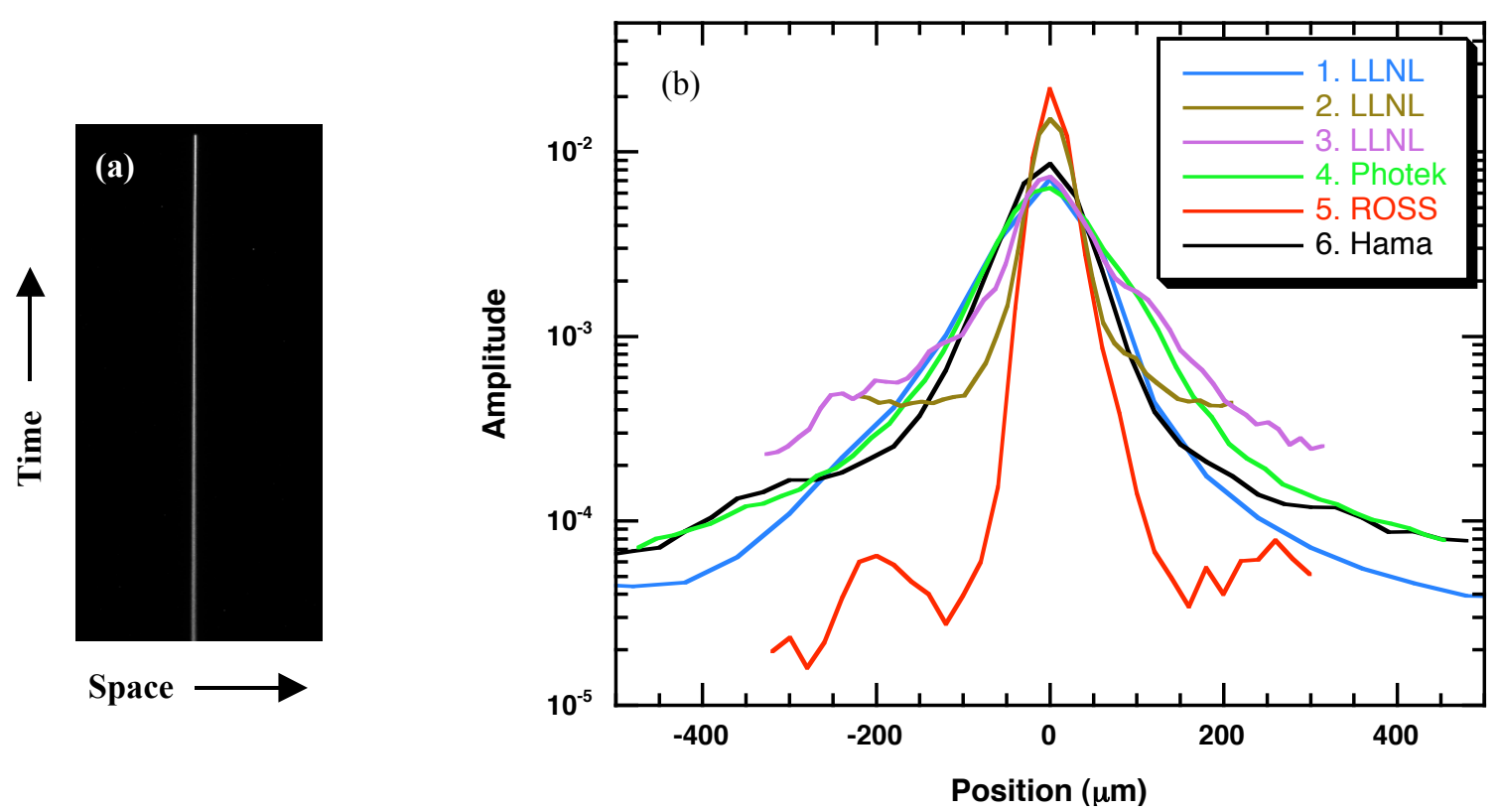

Figure 3. Line spread function. (a) Example of LSF data image. (b) Overlay of LSFs for six streak camera configurations

Each calculated CTF is verified with a couple of CTR measurements (typically at 4,6 , and $10 \mathrm{lp} / \mathrm{mm}$. Each measurement is made using a Ronchi ruling mask placed along the streak camera slit and illuminated by a low level, collimated laser beam (see example in Fig. 5). Analysis consists of first determining envelope functions for the peaks $\operatorname{Max}(\mathrm{x})$ and for the valleys $\operatorname{Min}(\mathrm{x})$ of the spatial lineout. Then the CTR is calculated from these functions as:

$$
\operatorname{CTR}(x)=[\operatorname{Max}(x)-\operatorname{Min}(x)] /[\operatorname{Max}(x)+\operatorname{Min}(x)]
$$

This technique allows calculation of the CTR without requiring a spatially uniform illumination of the modulation mask.

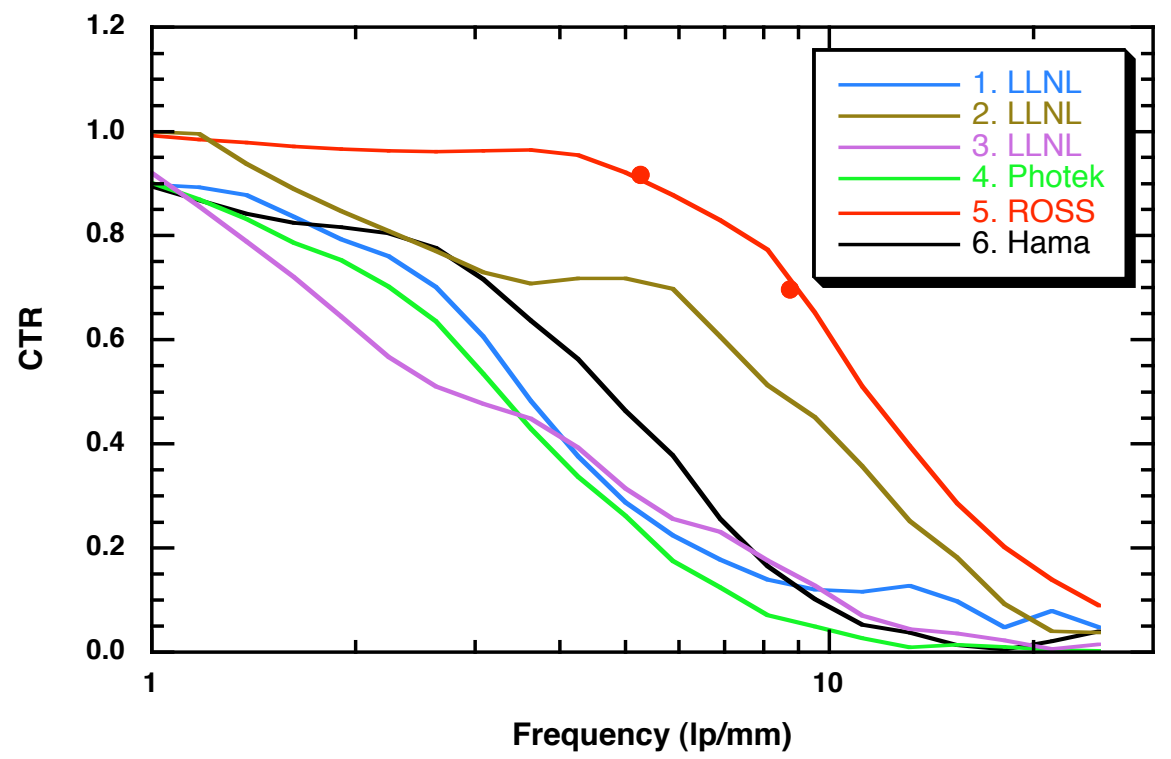

Figure 4. Contrast transfer functions (CTF) plotted for six streak camera configurations. Dots on ROSS curve are Ronchi ruling measurements made to confirm the CTF calculation. 


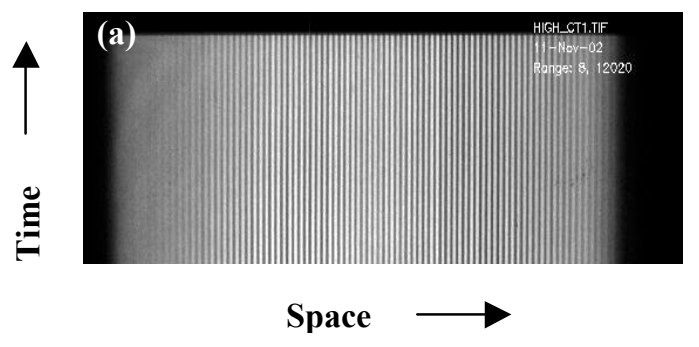

Figure 5. Sample Ronchi ruling CTR measurement for RCA C73435 (configuration 2): (a) Raw image of $4 \mathrm{lp} / \mathrm{mm}$ with $3 \times 3$ super-pixels, (b) Spatial intensity profile of (a), and (c) CTR calculated from intensy profile in (b).

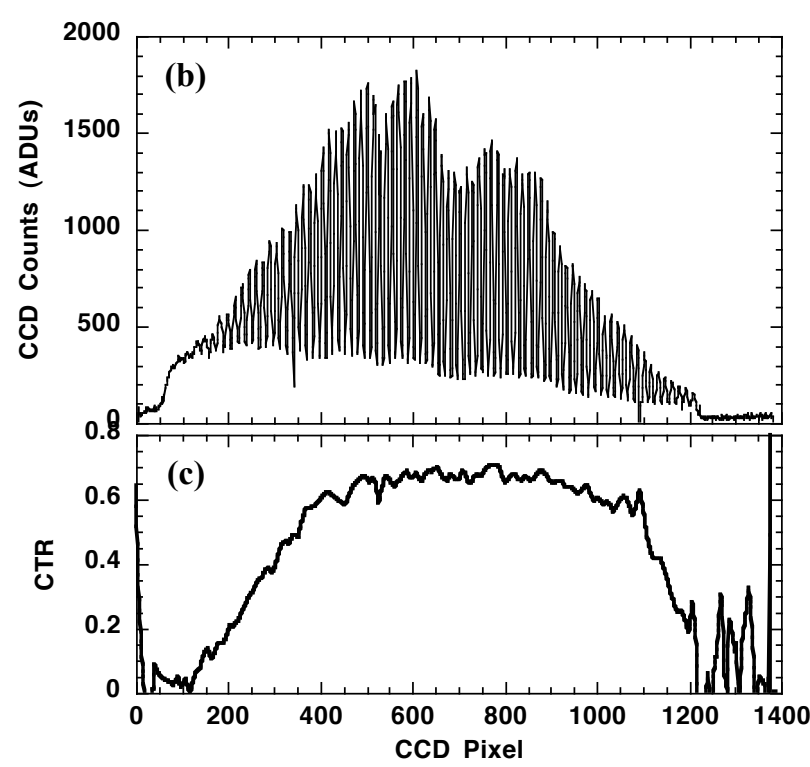

Ronchi ruling measurements are also made at higher input intensities to characterize the high current operation of the tube. With CTR measurements made at varying input signal intensities, camera response can be characterized as a function of tube current. For low currents the camera remains linear and the CTR observed matches the CTR calculated from the LSF. As input signal intensity increases, streak camera components eventually saturate and go nonlinear causing a loss in spatial resolution. For the first five camera configurations, loss of temporal resolution occurs at lower camera currents than does loss of spatial resolution.

Data were also recorded that allow spatial resolution to be mapped as a function of spatial position across an entire streak camera image. The data are recorded using the same mask used for magnification measurements, 10- $\square \mathrm{m}$ openings spaced $1.5 \mathrm{~mm}$ apart. The fwhm (in super-pixels) is determined at various spatial positions throughout the image and plotted as a contour map of fwhm versus position. The contour plots in Fig. 6 show the position dependence of the spatial resolution for the ROSS camera with 1000 - and $100-\square \mathrm{m}$ temporal slits.
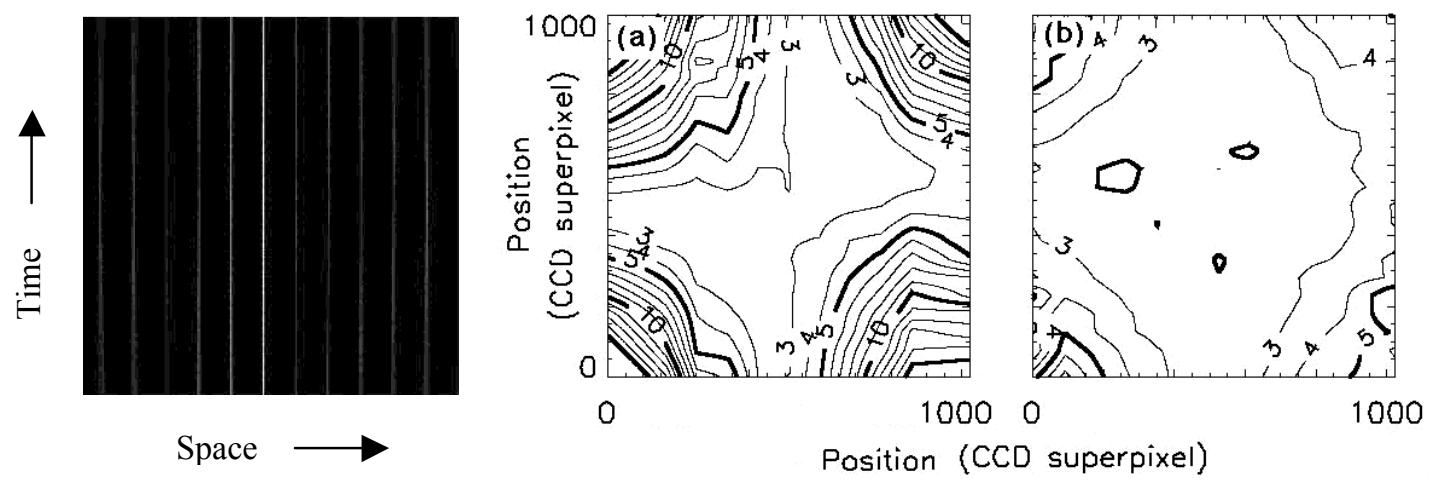

Figure 6. Spatial resolution versus position in streak camera image. (a) Streak camera image of 10 $\square \mathrm{m}$ slits spaced $1.5 \mathrm{~mm}$ apart. Contour plots show spatial resolution for the P510 with (b) $1 \mathrm{~mm}$ and (c) $100 \square \mathrm{m}$ wide input slits. Contour levels are signal fwhm given in super-pixels (2x2 binning). 

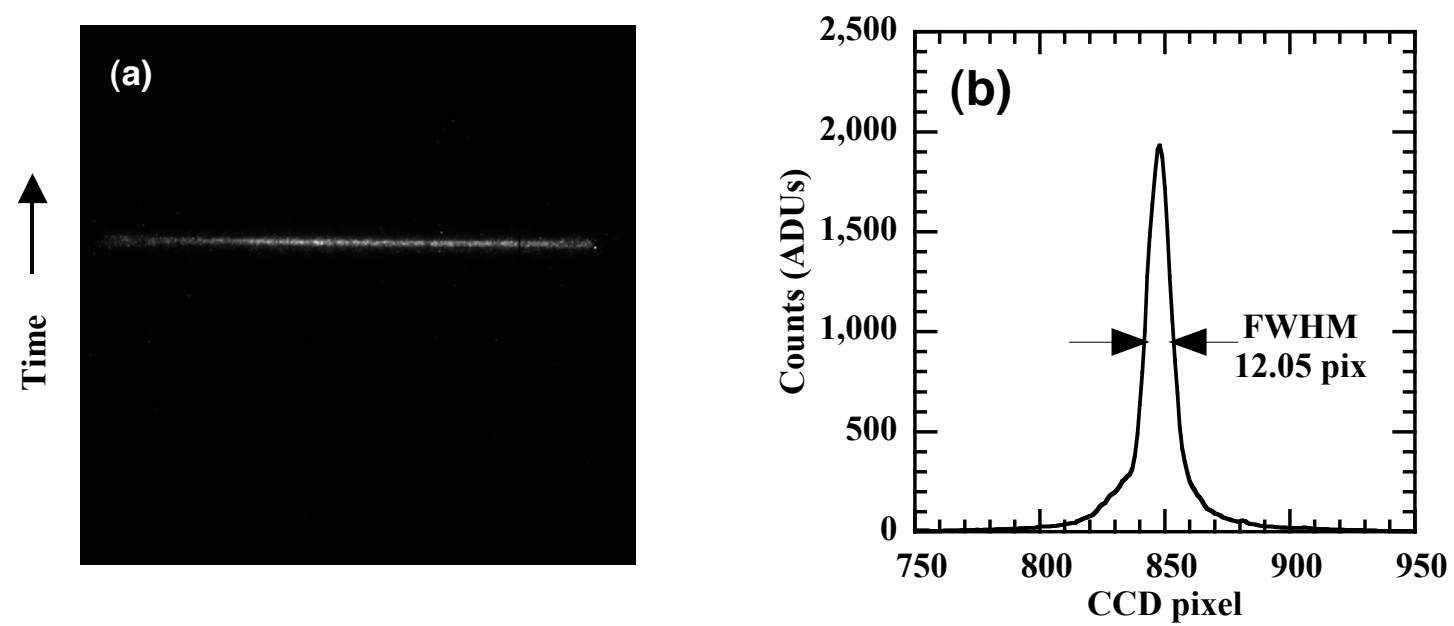

Figure 7. Static test provides estimate for temporal resolution. (a) Raw image recorded with deflection plates grounded (i.e. no sweep), $3 \times 3$ binning, configuration 2 with RCA C73435. (b) Lineout taken in the temporal direction of image in (a).

\subsection{Temporal Resolution}

A simple static test provides an excellent estimate for a streak camera's temporal resolution. For this measurement, the deflection plates are grounded and the tube illuminated with either a laser pulse or a DC light source to produce a nonswept line image like the one in Fig. 7(a). The fwhm $\square x$ of the static line represents the best temporal resolution (in pixels) possible with the camera. To form a good estimate for streak camera temporal resolution $\square \mathrm{t}_{\mathrm{sc}}$, simply multiply $\square \mathrm{x}$ by the dwell time $\mathrm{D}$ (ps/pixel) of the sweep and add the result in quadrature with the transient time spread of the photoelectrons traveling through the tube:

$$
\square \mathrm{t}_{\mathrm{sc}}=\left[(\square \mathrm{x} * \mathrm{D})^{2}+\left(\square \mathrm{t}_{\mathrm{tr}}\right)^{2}\right]^{1 / 2}
$$

Most of the transient time dispersion is caused by variations in initial electron velocities near the photocathode. For the Photonis P510 and the RCA C73435, $\square \mathrm{t}_{\mathrm{tr}}$ ranges from $\sim 6$ to $10 \mathrm{ps}$ depending on photocathode type, wavelength of the light, and electric field between photocathode and extraction grid.

Temporal resolution was checked with a 44 ps laser pulse recorded at three different sweep speeds: a fast sweep, an intermediate sweep, and a slow sweep. At all three sweep speeds, the measured pulse width matches that expected from static line width calculation:

$$
\square \mathrm{t}_{\text {est }}=\left[\left(\square \mathrm{t}_{\mathrm{sc}}\right)^{2}+\left(\square \mathrm{t}_{\mathrm{laser}}\right)^{2}\right]^{1 / 2}
$$

Table 3 show the measured temporal width matches the estimated response at all sweep speeds for two LLNL camera configurations. The measured signal width is dominated by the laser pulse width at fast sweep speeds; at slow sweeps, the measured signal width is limited by the static line width. The estimated and measured time responses to a 44-ps wide laser pulse show good agreement indicating a good estimate for the camera temporal resolution. All configurations with a slotted extraction grid had similar performance. Good performance from the Hamamatsu camera requires a slit width $<100 \square \mathrm{m}$. 


\begin{tabular}{|c|c|c|c|c|c|}
\hline$\square \mathrm{x}$ (pix) & Sweep Card & $\mathrm{D}$ (ps/pix) & $\square \mathrm{t}_{\mathrm{sc}}(\mathrm{ps})$ & $\square \mathrm{t}_{\text {est }}$ (ps) & $\square \mathrm{t}_{\text {meas }}(\mathrm{ps})$ \\
\hline \multirow{3}{*}{$\begin{array}{l}12 . \mathrm{LLNL} \\
\text { 2. }\end{array}$} & $1 \mathrm{x}$ & 0.6 & 12 & 46 & 45 \\
\hline & $3 x$ & 2.4 & 30 & 54 & 54 \\
\hline & 10x & 6.9 & 83 & 94 & 93 \\
\hline \multirow{3}{*}{$\begin{array}{l}13 \\
\text { 3. LLNL }\end{array}$} & $1 \mathrm{x}$ & 0.6 & 13 & 46 & 43 \\
\hline & $3 x$ & 2.4 & 33 & 55 & 58 \\
\hline & $10 \mathrm{x}$ & 6.9 & 90 & 100 & 101 \\
\hline
\end{tabular}

\subsection{Background Measurements}

Background images provide estimates for three basic system performance characteristics: read noise, dark current noise, and signal threshold. The manufacturer delivers the CCD readout with a specification sheet that lists the CCD read noise and dark current noise for its various gain settings. Background images allow us to quickly verify the manufacturers measurements and determine if any additional noise is introduced by other streak camera components. The standard deviation of the measured background $\square_{\text {noise }}$ is the quadrature sum of the dark current noise $\square_{\text {dark }}$ and the read noise $\square_{\text {read }}$. Because the dark current noise is proportional to image area and the read noise is independent of image area, background measurements made with different binning configurations can be used to determine the read noise and dark current noise. For example, if $2 \times 2$ and $3 \times 3$ pixel binning are considered, then the following set of three equations with three unknowns can be written:

$$
\begin{aligned}
& \square^{2}{ }_{\text {noise } 2 \times 2}=\square^{2} \text { dark } 2 \times 2+\square^{2} \text { read } \\
& \square_{\text {noise 3x3 }}^{2}=\square_{\text {dark } 3 \times 3}^{2}+\square_{\text {read }}^{2} \\
& \square_{\text {dark } 2 \times 2}^{2 \times}=(4 / 9) \square_{\text {dark } 3 \times 3}^{2}
\end{aligned}
$$

Thus, simply measuring the standard deviation of background images recorded with $2 \times 2$ and $3 \times 3$ binning allows calculation of dark current and read noise. Typically the average of four background images are used for each mesurement.

Some care must be taken in interpreting background images. Often, a background image will have a noticeable tilt because the last pixel read accumulates significantly more dark current than the first pixel read. The tilt adds to the standard deviation in the background image. There are several ways to handle the effect of the tilt. For this work, background images were recorded and smoothed with a large (11x11) boxcar filter. The raw image minus the filtered image forms a difference image containing only the noise centered at 0 . This is shown in the histogram for the difference image presented in Fig. 8. This technique gives results nearly identical to the more common method based on analyzing the difference between two background images.

The measurements show that the dark current noise and read noise for all camera configurations comes from the CCD and a negligible amount comes from the streak tube and MCP IIT. Measured read noise of 8.52 and $10.96 \mathrm{e}^{-}$for SI800 CCD gains of 0.43 and $2.31 \mathrm{e}^{-} / \mathrm{ADU}$ match the manufacturers measurements of

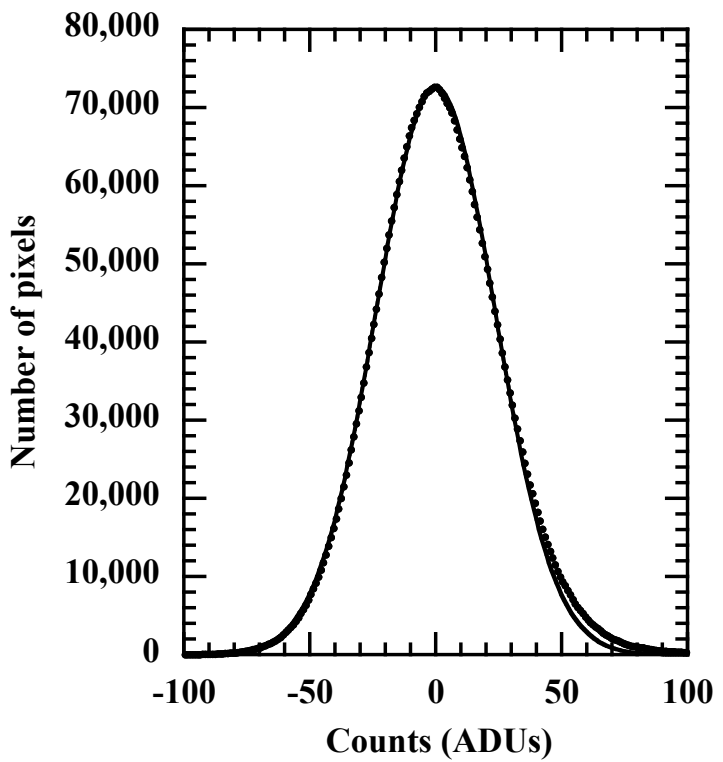

Figure 8. Histogram of pixels in measured difference image with $2 \times 2$ binning (SI-800). Solid line is Gaussian fit to data points, $\square_{\text {meas }} 2 \times 2$ $=23.61 \mathrm{ADUs}\left(10.15 \mathrm{e}^{-}\right)$. 
7.67 and $10.34 \mathrm{e}^{-}$, respectively. Dark current noise is independent of CCD gain. For 2-second exposures, dark current noise in $2 \times 2$ bins was 5.67 and $5.80 \mathrm{e}^{-}$.

Streak camera image noise determines the smallest signal or threshold that can be detected and sets a lower limit for the camera's dynamic range. Different groups and manufacturers use somewhat different criteria for this value ranging from $\square_{\text {noise }}$ to $5 \square_{\text {noise }}$. For this report we use the conservative value of $5 \square_{\text {noise }}$.

\subsection{System Sensitivity Measurements}

Full streak camera system sensitivity measurements are important for understanding dynamic range. In section 3.1 we discussed the relationship between incident optical energy and streak tube current. To fully understand streak camera performance, it is necessary to relate streak tube current to the observed output signal intensity. Full system sensitivity

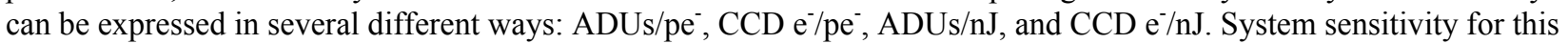
work is measured by illuminating a $3-\mathrm{mm}$ by $0.5 \mathrm{~mm}$ region of the photocathode with a 18 -ns long, $0.53 \square \mathrm{m}$, square pulse of known energy and recording an image. The number of ADUs in the recorded signal divided by the incident energy generating the signal gives the ADUs/nJ. Using the photocathode sensitivity (Amps/Watt) and the CCD gain (ADUs/CCD e ) allows conversion of this value to other units. Table 4 contains a summary of the system sensitivities for the streak camera configurations examined for this article.

\begin{tabular}{|c|c|c|c|c|}
\hline Configuration & 1. LLNL & 2. LLNL & 3. LLNL & 5. ROSS \\
\hline ADUs/pe- & 25 & 17.64 & 1,193 & 93 \\
\hline CCD e-/pe- & 500 & 7.59 & 2,791 & 101 \\
\hline ADUs/nJ & $3.12 \times 10^{9}$ & $2.2 \times 10^{9}$ & $1.49 \times 10^{11}$ & $1.45 \times 10^{10}$ \\
\hline CCD e-/nJ & $6.44 \times 10^{10}$ & $9.4 \times 10^{8}$ & $3.49 \times 10^{11}$ & $1.58 \times 10^{10}$ \\
\hline
\end{tabular}

\subsection{Dynamic Range}

Dynamic range is relatively simple concept: the maximum observable signal divided by the minimum observable signal. Unfortunately, problems quickly arise with the definitions used for the minimum and maximum observable signals. Are the signals quanta limited? Is the dynamic range quoted per CCD pixel, per CCD resolution element ( $2 \times 2$ pixel array), or per streak camera resolution element (which depends on both spatial and temporal resolution)? For this work, the dynamic range is presented per streak camera resolution element. The results can be converted to another basis if desired.

The minimum signal $\mathrm{S}_{\min }$ is conservatively taken as $5 \square_{\text {noise }}$ (see Section 3.2). A signal of this amplitude will normally be detectable above the background noise. Since dynamic range is calculated per resolution element for this article, the noise must also be calculated per resolution element rather than per CCD pixel. If a resolution element contains N pixels, the noise sums across the pixels in the resolution element and the minimum observable signal is given by $\mathrm{S}_{\min }=$ $5 \square$ noise $\mathrm{N}^{1 / 2}$. If a single photoelectron produces more signal than $\mathrm{S}_{\min }$, then the system is quantum limited and the signal from a single photoelectron should be used as $\mathrm{S}_{\min }$.

The maximum signal $\mathrm{S}_{\max }$ used for dynamic range calculations is somewhat more difficult to define. For this article, we use the signal at which the temporal pulse broadens by $20 \%$ independent of the broadening mechanism. A definition for the maximum signal based on temporal broadening depends on sweep speed and works best when the input pulse width is short relative to the streak camera resolution. When the streak tube is the limiting element, temporal broadening can be correlated with the C-L space-charge-limited current.

To determine the maximum signals to be used for dynamic range calculations, temporal signals were recorded at various sweep speeds and signal amplitudes. For this work, 44-ps fwhm laser pulses were recorded with nominal 1, 3, and 10-ns streak camera windows. An example is shown in Fig. 9. Analysis typically uses two more views of the data to help determine what actually limits the maximum signal: peak signal versus input energy and total signal versus input energy. 


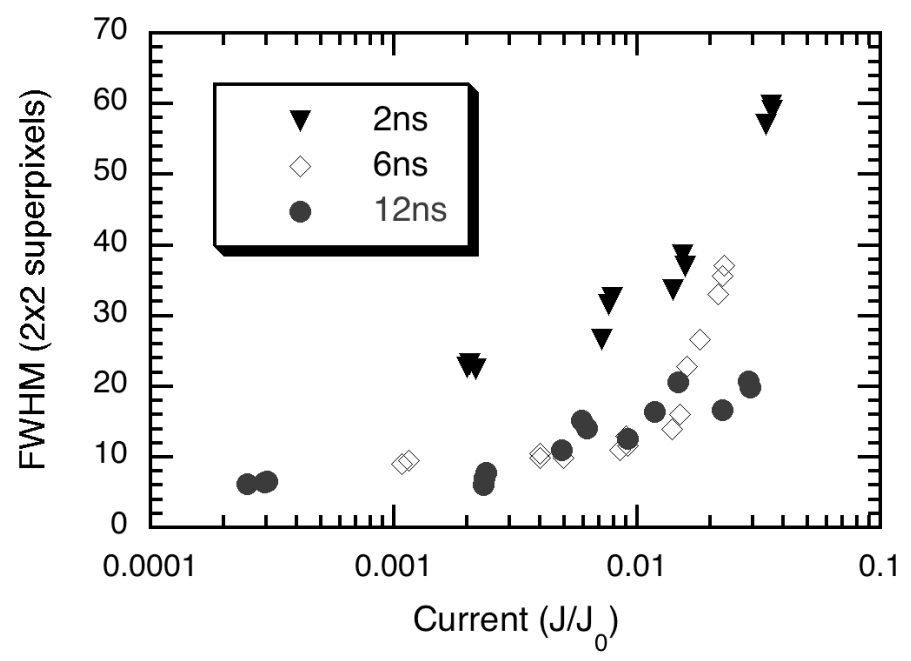

Figure 9. Temporal broadening of 44-ps laser pulse versus P510 streak tube current density for 2,6 , and $12 \mathrm{~ns}$ sweep speeds. $\mathrm{J}_{0}=2.2 \mathrm{~A} / \mathrm{cm}^{2}$.

With no IIT present, the large current density in the streak tube needed to produce an output signal causes temporal broadening. At all three sweep speeds the temporal signal broadens and the peak amplitude of the signal drops below a linear curve proportional to the input energy. But even so, the total integral of the signal remains linear with input signal indicating no loss of streak tube current, just a loss in resolution. This is not the case for the standard LLNL camera where the MCP IIT begins to run out of charge. ${ }^{6}$

Once the $20 \%$ broadening point of the signal is found, then the maximum signal per CCD pixel can be calculated in either ADUs or photoelectrons. The maximum signal per camera resolution element is then $\mathrm{N}$ times this value. For high gain systems in which there are many ADUs per photoelectron, the minimum signal in a dynamic range calculation must be replaced by the equivalent signal of one photoelectron. This can be much greater than the system noise of 5 $\square_{\text {noise }}$.

\section{DISCUSSION}

Section 3 describes the characterization methods used for evaluating streak camera and streak tube performance. Characteristics for the six streak camera configurations examined in this study are summarized in Table 5.

Three of the camera configurations used IITs to enhance their output signal intensity. Two of these configurations used the obsolete RCA C73435 and one used the Hamamatsu N8059. The three LLNL camera configurations demonstrate the effect of the MCP IIT on system resolution and show that the RCA tube actually has much better spatial resolution than it achieves in the LLNL "blue box" configuration. In the "blue box" configuration (\#1) the LSF is $120 \square \mathrm{m}$ fwhm. When the MCP IIT is removed and the streak tube is directly coupled to a large format CCD (configuration \#2) the LSF improves to $50-\square \mathrm{m}$ fwhm. When the camera is reconfigured with the MCP IIT and a direct-coupled CCD readout (configuration \#3), the LSF again broadens to $100-\square \mathrm{m}$ fwhm. These three configurations demonstrate the significant degradation in image resolution caused by the MCP IIT. Elimination of the MCP IIT improves spatial resolution by a factor of 2.4 and allows the system to be limited by the C 73435 response. The Hamamatsu camera was equipped with an image booster (config. \#6). It is speculated that this image intensifier stage degrades the spatial performance and masks the true capabilities of the Hamamatsu tube. The Photonis P510 streak tube (configuration \#5) shows a slightly better spatial resolution than the C73435 (configuration \#2). It is unknown why the Photek ST-Y (configuration \#4) has such poor spatial resolution $(130-\square \mathrm{m}$ fwhm $)$ as it is configured in the same manner as the high performance LLNL camera (configuration \#2) and the Photonis P510 (configuration \#5). Only the ROSS configuration has eliminated the low level 
wings of the LSF. All configurations with IITs show low-level wings caused by veiling glare (see Fig. 3). The excellent LSF of the ROSS results in a nearly ideal CTF with a $50 \%$ CTR at $10 \mathrm{lp} / \mathrm{mm}$ and a limiting visual CTR $25 \mathrm{lp} / \mathrm{mm}$ (see Fig. 4).

\begin{tabular}{|c|c|c|c|c|c|c|}
\hline Configuration & 1. LLNL & 2. LLNL & 3. LLNL & 4. Photek & 5. ROSS & 6. Hama \\
\hline Streak Tube & $\begin{array}{c}\text { RCA } \\
\text { C73435 }\end{array}$ & $\begin{array}{c}\text { RCA } \\
\text { C73435 }\end{array}$ & $\begin{array}{c}\text { RCA } \\
\text { C73435 }\end{array}$ & $\begin{array}{l}\text { Photek } \\
\text { ST-Y }\end{array}$ & $\begin{array}{c}\text { Photonis } \\
\text { P510 }\end{array}$ & $\begin{array}{c}\text { Hamamtsu } \\
\text { N8059 }\end{array}$ \\
\hline Image Intensifier & YES & $\mathrm{NO}$ & YES & $\mathrm{NO}$ & $\mathrm{NO}$ & YES \\
\hline Large format CCD & $\mathrm{NO}$ & YES & YES & YES & YES & YES \\
\hline Field off View $(\mathrm{mm})$ & 20.2 & 20.2 & 20.2 & 19.0 & 20.5 & 13.0 \\
\hline Magnification & 1.45 & 1.45 & 1.45 & 1.30 & 1.35 & $1.00 *$ \\
\hline \multicolumn{7}{|c|}{ Spatial Resolution - referred to streak tube photocathode } \\
\hline LSF fwhm $(\square \mathrm{m})$ & 120 & 50 & 100 & 130 & 40 & 90 \\
\hline CTR $(50 \%)$ & 3.8 & 8.0 & 2.2 & 3.3 & 10.0 & 5.0 \\
\hline \multicolumn{7}{|l|}{ Temporal Resolution } \\
\hline Static width (super-pixels) & $3(3 \times 3)$ & $12(3 \times 3)$ & $12(3 \times 3)$ & $4.5(3 \times 3)$ & $4(2 \times 2)$ & $4.5(2 \times 2)$ \\
\hline $1 \mathrm{~ns}$ window & 12 & 12 & 13 & 12 & 12@2ns & $12 @ 2 \mathrm{~ns}$ \\
\hline 3 ns window & 29 & 30 & 33 & & 22@5ns & 48@5ns \\
\hline 10 ns window & 80 & 83 & 90 & & 45@11ns & 83 \\
\hline \multicolumn{7}{|l|}{ System Sensitivity } \\
\hline ADUs/pe & 25 & 17.65 & 1193 & & 101 & \\
\hline $\mathrm{CCD}$ e / pe & 500 & 7.6 & 2791 & & 108 & \\
\hline \multicolumn{7}{|l|}{ Noise (electrons) } \\
\hline$(1 \times 1)$ & & & & & 5.1 & \\
\hline$(2 \times 2)$ & & 8.5 & 8.5 & & 6.0 & \\
\hline$(3 \times 3)$ & 25.0 & 11.0 & 11.0 & & 6.7 & \\
\hline$(4 \times 4)$ & & & & & 7.9 & \\
\hline Noise (5ロ) per res element & 425 & 150 & 235 & & 85 & \\
\hline \multicolumn{7}{|l|}{ Dynamic Range } \\
\hline Super-pixels / Res element & 18 & 12 & 30 & & 8 & \\
\hline $1 \mathrm{~ns}$ window & 1563 & 27 & 244 & & 500 & \\
\hline 3 ns window & 1024 & 86 & 163 & & 1150 & \\
\hline $10 \mathrm{~ns}$ window & 960 & 249 & 153 & & 2450 & \\
\hline & & & & & & \\
\hline
\end{tabular}

The input field-of-view (FOV) (referred to the streak tube photocathode) is nearly identical at just over $20 \mathrm{~mm}$ for the RCA, Photonis, and Photek tubes. The Hamamatsu tube has only a 13-mm FOV. This measurement is made with collimated light entering the streak camera input slit through a Ronchi ruling mask. When input light at the Hamamatsu input slit is diffuse, the FOV increases to $\sim 17 \mathrm{~mm}$. It was found that the Hamamatsu camera has a 13-mm diameter input lens that limits the FOV. With a diffuse image at the slit, there is significant vignetting of the input signal at the outer ends of the slit. 


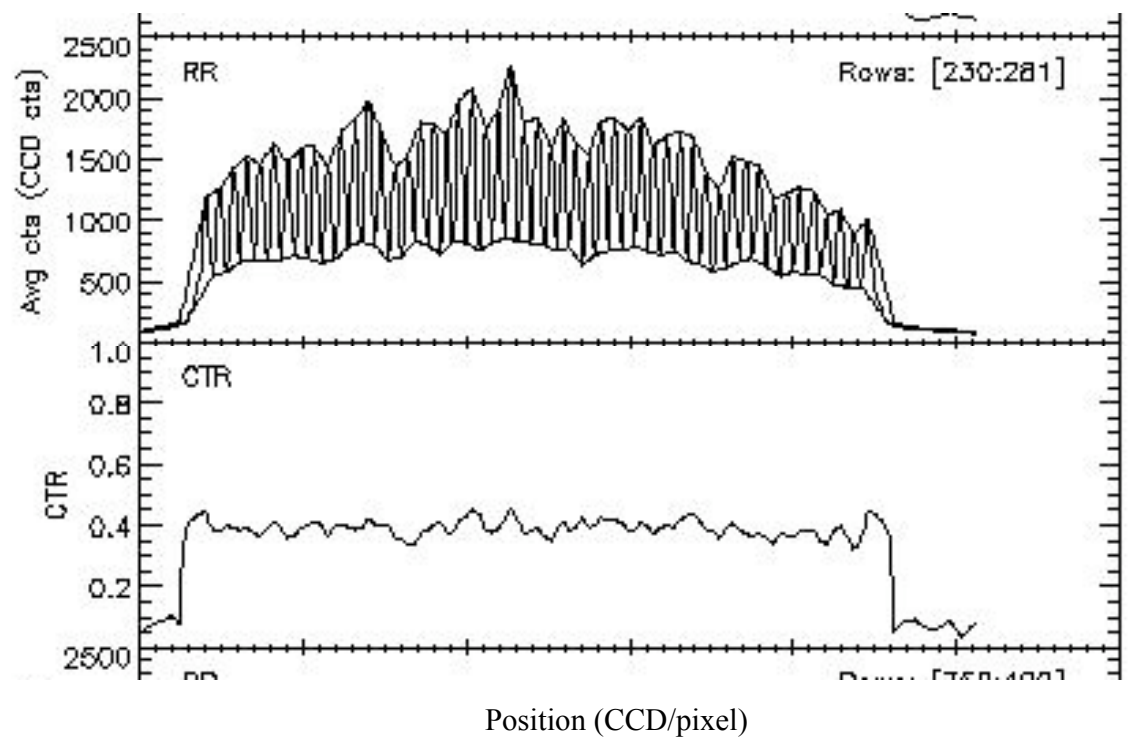

Figure 10. The Hamamatsu N8059 shows extremely uniform spatial response across its entire output image as shown with this Ronchi ruling measurement and corresponding CTR.

One significant characteristic of the Hamamatsu camera is its uniformity of resolution across the entire output image. This can be seen in the Ronchi ruling image presented in Fig, 10. Here the CTR is flat across the entire image. This was not the case for the RCA tube (see Fig. 5c) or the Photonis tube (see Fig. 6). Note, the Hamamatsu tube is evaluated over a FOV of $13 \mathrm{~mm}$ while the other tubes were evaluated over a FOV of $20 \mathrm{~mm}$.

The internal spatial magnification of the three electrostatic focused tubes ranges from 1.3 to 1.45 . The magnetically focused tube has a magnification closer to 1 .

Temporal resolution is found to be less dependent than spatial resolution on the streak camera configuration. It is proportional to the static line width except for the fastest sweep speeds. For LLNL standard operation, the electrostatic focused tubes use 1-mm wide slits (in temporal direction). For the RCA C73435 tube, all three configurations produced nearly identical temporal resolutions. In wide slit operation at the $2 \square$ wavelength, the tube has somewhat poorer focusing ability in the temporal direction than it has in the spatial direction making the degradation by the MCP IIT relatively unimportant. Slight improvement in temporal resolution at the expense of system sensitivity can be obtained by reducing the slit width. ${ }^{11}$ Measurements with the Hamamatsu camera were made with a $100-\square \mathrm{m}$ wide slit. Wider slits made the Hamamatsu temporal resolution significantly worse. There is slight improvement in time resolution at slower sweep speeds when narrower slits $(<100 \square \mathrm{m})$ are used.

The systems vary significantly in their sensitivity to a single photoelectron emitted from the streak tube photocathode. The three electrostatic focused tubes have electron acceleration to $15 \mathrm{kV}$, the Hamamatsu accelerates the electrons to 10 $\mathrm{kV}$. System sensitivity ranged from 7.6 to $2791 \mathrm{CCD}$ electrons per photoelectron from the streak tube photocathode, or equivalently 17.64 to 1193 ADUs per photoelectron. This wide range in sensitivity is dominated by camera configuration rather than by the streak tube characteristics. (See configurations 1,2, and 3 which all use the C73435.) What is most important is the ratio of signal per photoelectron to the noise per resolution element. Two of the cameras have been optimized in this respect (configurations \#1 and \#5). In these two cases the signal per photocathode photoelectron is slightly greater $(\sim 20 \%)$ than the noise in a resolution element. This places the lower end of the camera range at the ideal spot, just below the threshold for detecting a single event. 
In the case of configuration \#2 the system lacks enough sensitivity for good dynamic range. It takes 20 photoelectrons to reach the detection threshold. This reduces the overall dynamic range for this system by a factor of 20 below the C73435's capability. With the addition of the MCP IIT in configuration \#3, the signal produced is a factor of 12 above the noise threshold. In this case the signal is quanta limited. That is, a single photoelectron produces way more CCD electrons than the detection threshold (2791 to 235). Here the lower end of the CCD range is wasted.

The dynamic range varies considerably between the three camera configurations. Dynamic range results are reported per streak camera resolution element (spatial width times temporal width in pixels). Configuration \#2 without the IIT lacks enough amplification to clearly see a single photoelectron. This results in the low dynamic range. An improvement in the relatively poor QE of $15 \%$ for this particular CCD to $75 \%$ would boost all the dynamic range values by a factor of 5 . An additional factor of two in noise reduction would bring the total dynamic range improvement to a factor of 10 . This would allow the camera to come within a factor of two of the C73435's C-L current limited dynamic range potential. Configuration 3 has the opposite problem. The MCP IIT gain is too high which results in a very large signal for a single photoelectron. With the high gain, the system is linear until the CCD reaches its upper limit of 32,000 ADUs, but the minimum observable signal of $2791 \mathrm{CCD}$ electrons per photoelectron is well above the signal needed to simply surpass the noise. The large dynamic range that is associated with configuration 1 is simply a result of work done in the late 1980s when slit width, MCP gain, and other operating parameters were optimized. The same is true of the Photonis P510 in the ROSS configuration. Components and their operating points have been carefully selected to optimize camera performance. We did not acquire all the data necessary to determine the dynamic range of the Photek and Hamamatsu systems.

In summary, the elimination of the IIT used in the "blue box" and the addition of the large-format CCD show great promise. Clearly, spatial resolution improves with the elimination of the MCP IIT. In the data reported here, system gain of the ROSS configuration without the IIT is nearly ideal and full dynamic range of the the P510 streak tube is achieved. The large-format CCD used in the ROSS has been optimized. Had the E2V CCD and its fiber optic coupler been used with configuration \#2, the RCA C73435 dynamic range would improve by a factor of 5 to 10 .

\section{ACKNOWLEDGMENT}

This work was performed under the auspices of the U.S. Department of Energy by the University of California Lawrence Livermore National Laboratory under contract No. W-7405-Eng-48.

\section{REFERENCES}

1. S. W. Thomas, J. W. Houghton, G. R. Tripp, and L. W. Coleman, "The LLL compact 10 ps streak camera-1974 update," Proc. of $11^{\text {th }}$ Int'1 Cong. on High Speed Photography, London, p. 101 (1974).

2. R. A. Lerche, "Timing between streak cameras with a precision of $10 \mathrm{ps,"} \mathrm{Proc.} \mathrm{of} \mathrm{SPIE} \mathrm{Conference} \mathrm{on}$ Ultrahigh- and High-Speed Photography, Videography, Photonics, and Velocimetry '90, SPIE Vol. 1346, pp. 376-383 (1990).

3. D. T. Attwood, R. L. Kauffman, G. L. Straddling, H. L. Medecki, R. A. Lerche, L. W. Coleman, E. L. Pierce, S. W. Thomas, D. E. Campbell, J. Noonan, G. R. Tripp, R. J. Schnetz, and G. E. Phillips, "Picosecond X-Ray Measurements from $100 \mathrm{eV}$ to $30 \mathrm{keV}$ ", Proc. of XIV International Congress on High Speed Photography and Photonics, Moscow (1980).

4. R. A. Lerche, D. W. Phillion, and G. W. Tietbohl, "Neutron detector for fusion reaction-rate measurements," Proc. of SPIE Conference on Ultrahigh- and High-Speed Photography, Videography, and Photonics, '93, SPIE Vol. 2002, pp. 153-161 (1993).

5. B. A. Jones, "A lens coupled streak camera readout system utilizing a thermoelectric cooled CCD," Conf. on High Speed Photography, Videography and Photonics III, SPIE Vol. 569, pp 189-193 (1985).

6. Dick Lerche and Roger Griffith, "CCD Replacement for LLNL Optical Streak Cameras," Lawrence Livermore National Laboratory NIF memo, NIF-0004058 (1997).

7. J. D. Wiedwald and R. J. Hertel, "Veiling Glare in the ITT F4113 Image Intensifier", Proc. of SPIE Conference on High Speed Photography, Videography, and Photonics VI, SPIE Vol. 981, pp. 154-160 (1988). 
8. Richard A. Lerche, David S. Andrews, Perry M. Bell, Roger L. Griffith, Joseph W. McDonald, Peter Torres III, and Gene Vergel de Dios, "Streak camera performance with large-format CCD readout", Proc. Int. Soc. Opt. Eng. 5210, 1 (2004).

9. R.A. Lerche, J.W. McDonald, R.L. Griffith, G.Vergel de Dios, D.S. Andrews, A.W. Huey, P.M. Bell, and O.L. Landen, "Preliminary performance measurements for a streak camera with a large-format direct-coupled CCD readout", Rev. Sci. Instrum., 75 (10), pp. $4042-4044$ (2004).

10. J. D. Wiedwald and R. A. Lerche, "Streak camera dynamic range optimization", Proc. of Conference on High Speed Photography, Videography, and Photonics V, SPIE Vol 832, pp. 2275-282 (1987).

11. R. A. Lerche and R. L. Griffith, "Resolution limitations and optimization of the LLNL streak camera focus," Proc. of Conference on High Speed Photography, Videography, and Photonics V, SPIE Vol 832, pp. 266-274 (1987).

12. R. Kalibjian and G. G. Peterson, "Analysis of space charge in photonic tubes", J. Appl. Phys., 54, pp. 4295-4301 (1983). 